\title{
PARAMETER IDENTIFICATION OF THE BASSET FORCE ACTING ON PARTICLES IN FLUID FLOW INDUCED BY THE OSCILLATING WALL
}

\author{
Ivan Pavlenko ${ }^{1}$, Oleksandr Liaposhchenko ${ }^{2}$, Jan Pitel $^{3}$, Vsevolod Sklabinskyi ${ }^{2}$ \\ ${ }^{l}$ Department of General Mechanics and Machine Dynamics, Sumy State University \\ Sumy, Ukraine \\ ${ }^{2}$ Processes and Equipment of Chemical and Petroleum-Refineries Department \\ Sumy State University, Sumy, Ukraine \\ ${ }^{3}$ Institute of Manufacturing Management, Technical University of Kosice \\ Presov, Slovakia \\ i.pavlenko@omdm.sumdu.edu.ua,o.liaposhchenko@pohnp.sumdu.edu.ua, jan.pitel@tuke.sk \\ v.sklabinskyi@pohnp.sumdu.edu.ua
}

Received: 26 February 2019; Accepted: 3 July 2019

\begin{abstract}
The article is aimed at the development of the analytical approach for evaluating the parameters of the Basset force acting on a particle in two-dimensional fluid flow induced by the oscillating wall. By applying regression analysis, analytical expressions to determine complementary functions were established for evaluating the Basset force. The obtained dependencies were generalized using the infinite power series. As a result of studying the hydrodynamics of a two-phase flow, analytical dependencies to determine the Basset force were proposed for assessing its impact on particles of the dispersed phase in a plane channel with the oscillating wall. It was discovered that the Basset force affects larger particles. However, in the case of relatively large wavelengths, its averaged value for the vibration period is neglected. Additionally, the value of the Basset force was determined analytically for the case of relatively small wavelengths. Moreover, it was discovered that its impact can be increased by reducing the wavelength of the oscillating wall.
\end{abstract}

MSC 2010: 76T10, 65Z05

Keywords: hydrodynamics, vibration impact, velocity field, complementary functions, regression analysis, infinite power series

\section{Introduction}

There is a number of forces of different nature acts on fine particles of the dispersed phase in the multiphase flow under the vibration and acoustic fields. In this case, one of the most incompletely unexplored in terms of analytical calculation is the Basset force. For example, articles [1,2] show that this force affects the direction of particles drift in a standing wave. And the phenomenon of a particles drift for the dispersed phase is widespread in the technology of purification and coagulation. 
The dynamics of a single particle in a wave field is detailed and considered in the articles [3, 4]. Particularly, it is shown that there is a wave force acting upon the particle that predetermines the average acceleration of the particle for the period of oscillation. P.J. Westervelt showed that one of the reasons for the phenomenon of particles drift is the nonlinearity of the resistance distribution law [5]. This fact leads to the existence of higher harmonics in the solution of the Langevin equation [6] describing the stochastic motion of particles in a turbulent flow bounded by walls. Additionally, based on the Kolmogorov's Lagrangian similarity law [7], asymptotic expressions for turbulent energy dissipation are obtained, and expressions similar to Onsager reciprocal relations are defined for describing several non-equilibrium processes (e.g. heat transfer, diffusion) simultaneously occurring in a closed macroscopic environment and mutually affecting each other [8]. Moreover, the limiting transformation of the Langevin equation determines the equation of diffusion for the case of dispersed impurities in a flow with turbulence due to the walls.

Investigation of the stochastic turbulent motion of particles based on the Langevin equation contains the assumption that the acceleration of a liquid particle is a delta-correlated random process. In this case, the correlation function is conditionally expressed in terms of the Dirac delta function. The justification of the reliability of this assumption in the theory of turbulence is the experimentally confirmed fact that acceleration is determined by the small-scale "viscous" motions (relative to the Kolmogorov scale) $[9,10]$.

On the other hand, scientists pay sufficient attention to the study of particles drift in inhomogeneous wave fields, e.g. for the case of a periodic shock wave of the resonator. Particularly, L.V. King obtained the formula for radiation pressure [11]. As a result, aerosol particles in a flow with a relatively low density are drifting towards the antinodes. And conversely, relatively light particles are drifting to the wave nodes. Additionally, these formulas are generalized for the case of a compressible medium [12] and are experimentally confirmed [13].

The article [14] is devoted to studying the aerosol particles in a plane standing wave for the case of small Reynolds numbers and relatively low frequencies of the vibration impact. As a result, the theory of inclusion drift is proposed considering the Stokes, added mass and Basset forces.

The Basset force is one of the components of the resistance force acting upon particles in a viscous fluid. Generally, this force is determined by integrating the pressure function on the surface of the body. In this case, the pressure field is determined as a solution of the system of the Navier-Stokes and continuity equations [15]. After neglecting the local and convective inertia forces, the Stokes force is obtained for a particle of the spherical form in a fluid flow. This force is proportional to the relative velocity of the particle. Subsequently, Boussinesq and Basset independently obtained a more accurate analytical solution for the case of neglecting the convective forces of inertia only. As a result, the resistance force is obtained with the following three components: the force of added mass proportional to the relative acceleration of the particle in the flow, the Stokes force calculated previously, and the Basset force [16]: 


$$
F_{B}=\frac{3}{2} d_{p}^{2} \sqrt{\pi \rho \mu} \int_{0}^{t} \frac{d\left(v-v_{p}\right) / d t}{\sqrt{t-\theta}} d \theta,
$$

where: $d_{p}$ - particle diameter $[\mathrm{m}] ; \rho, \mu$ - density and dynamic viscosity of the medium, respectively $\left[\mathrm{kg} / \mathrm{m}^{3}\right]$ and $[\mathrm{Pa} \cdot \mathrm{s}] ; v, v_{p}$ - medium and particle velocities, respectively $[\mathrm{m} / \mathrm{s}] ; t$ - time; $\theta$ - integration variable as a time parameter $[\mathrm{s}]$.

Thus, the Basset force depends both on the vector of relative acceleration of the particle in the flow and on its values at all previous time moments. And now it is confirmed that for a given density of inclusions there is a limiting frequency, above which the wave force changes the sign. Moreover, it is proved that the Basset force substantially affects the limiting frequency.

Due to the abovementioned, despite a large number of studies of particles drift, the problem of determining the Basset force and its impact on fine particles of the disperse phase is an open issue. Consequently, the main aim of the research is to develop an approach for analytically calculating the parameters of the Basset force acting on particles in fluid flow induced by the oscillating wall.

\section{Research methodology}

\subsection{Previous approximation of the Basset force}

For relatively inert particles, when its relative velocity is insignificant compared to the flow velocity $\left(\left|v_{p}\right|<<|v|\right)$, the specific Basset force $f_{B}\left[\mathrm{~m} / \mathrm{s}^{2}\right]$ (per unit mass of particle $\left.m_{p}=\rho_{p} \cdot \pi d_{p}^{3} / 6[\mathrm{~kg}]\right)$ is determined by the following dependence:

$$
f_{B}=\frac{F_{B}}{m_{p}} \approx \frac{9}{\rho_{p} d_{p}} \sqrt{\frac{\rho \mu}{\pi}} \int_{0}^{t} \frac{d v / d t}{\sqrt{t-\theta}} d \theta .
$$

As it was obtained previously in articles [17-19], the vibration impact of an oscillating wall to the flow leads to a change of the particle velocity by a periodic law, which is a superposition of several components.

Due to the nonlinearity of the specific Basset force (2) in terms of the flow velocity, its value can be determined by adding the corresponding components for each of the harmonics. Considering the expression for the acceleration component of each harmonic:

$$
d v(t) / d t=a_{0} \sin \omega t
$$

for the amplitude $a_{0}$ and vibration frequency $\omega$, the following formula can be written:

$$
f_{B}=a_{0} C_{B} i_{s}(t, \omega),
$$

where the dimensional parameter of the Basset function is introduces $\left[\mathrm{s}^{-1 / 2}\right]$ :

$$
C_{B}=9 \sqrt{\rho \mu / \pi} /\left(\rho_{p} d_{p}\right)
$$


and the complementary function is:

$$
i_{s}(t, \omega)=\int_{0}^{t} \frac{\sin \omega \theta}{\sqrt{t-\theta}} d \theta
$$

Thus, the problem of determining the Basset force acting on a particle of the dispersed phase in two-phase flow is reduced to obtaining the approximate analytical expression for the complementary function $i_{s}(t, \omega)$.

It should be noted that the numerical integration of the right part of the expression (6) for a wide range of frequency values (from $0.01 \mathrm{rad} / \mathrm{s}$ to $10^{5} \mathrm{rad} / \mathrm{s}$ ) allows one to conclude that the evaluated function $i_{s}(t, \omega)$ can be presented with the phase shift $\pi / 4$ in the following form:

$$
i_{s}(t, \omega) \approx A(\omega) \sin (\omega t-\pi / 4),
$$

where $A(\omega)$ - amplitude-frequency function, which is tabulated in Table 1.

Table 1. Tabulation of the amplitude-frequency function

\begin{tabular}{|c|c|c|c|c|c|}
\hline No. & $\begin{array}{c}\text { Frequency } \\
\omega[\mathrm{rad} / \mathrm{s}]\end{array}$ & $\begin{array}{c}\text { Amplitude } \\
A\left[\mathrm{~s}^{1 / 2}\right]\end{array}$ & No. & $\begin{array}{c}\text { Frequency } \\
\omega[\mathrm{rad} / \mathrm{s}]\end{array}$ & $\begin{array}{c}\text { Amplitude } \\
A\left[\mathrm{~s}^{1 / 2}\right]\end{array}$ \\
\hline 1 & 0.01 & 18.2 & 8 & 100 & 0.18 \\
\hline 2 & 0.1 & 5.8 & 9 & 150 & 0.15 \\
\hline 3 & 1 & 1.76 & 10 & 200 & 0.13 \\
\hline 4 & 5 & 0.81 & 11 & 500 & 0.08 \\
\hline 5 & 10 & 0.57 & 12 & $10^{3}$ & 0.057 \\
\hline 6 & 25 & 0.36 & 13 & $10^{4}$ & 0.018 \\
\hline 7 & 50 & 0.25 & 14 & $10^{5}$ & 0.006 \\
\hline
\end{tabular}

Moreover, an asymptotic approximation of the function $A(\omega)$ to zero under the limitation $\omega \rightarrow \infty$, as well as its infinite growth under the limitation $\omega \rightarrow 0$ allows one to propose the following expression for the amplitude-frequency function:

$$
A(\omega)=c / \omega^{n} .
$$

where: $c$ - dimensionless coefficient, $n$ - power factor, which is equal to 0.5 due to the dimension of the amplitude-frequency function.

The dimensionless coefficient $a$ is determined using the regression analysis based on the least square method. In this case, the error function is the total quadratic deviation of values (8) for $n=0.5$ from the values tabulated in Table 1:

$$
R(c)=\sum_{i=1}^{N}\left(c / \sqrt{\omega_{i}}-A_{i}\right)^{2} \rightarrow \min ,
$$

where: $A_{i}-i$-th tabulated value of the amplitude-frequency function for the related frequency $\omega_{i}, N=14$ - total number of the tabulated data. 
Minimization of the error function by the procedure of finding its first derivative with respect to the argument $c$ :

$$
\frac{d R(c)}{d c}=2 \sum_{i=1}^{N}\left(\frac{c}{\sqrt{\omega_{i}}}-A_{i}\right) \frac{1}{\sqrt{\omega}}=2\left(c \sum_{i=1}^{N} \omega_{i}^{-1}-\sum_{i=1}^{N} \frac{A_{i}}{\sqrt{\omega_{i}}}\right)=0
$$

allows one to obtain the linear regression formula for the evaluated parameter $c$ :

$$
c=\sum_{i=1}^{N} A_{i} \omega_{i}^{-1 / 2} / \sum_{i=1}^{N} \omega_{i}^{-1}
$$

Substitution of values from Table 1 to the expression (11) allows one to estimate the dimensionless coefficient: $c=1.82$.

The accuracy of the proposed approach is graphically illustrated in Figure 1 by comparing the expression (8) for the parameters $c=1.82$ and $n=0.5$ with the numerical data presented in Table 1.

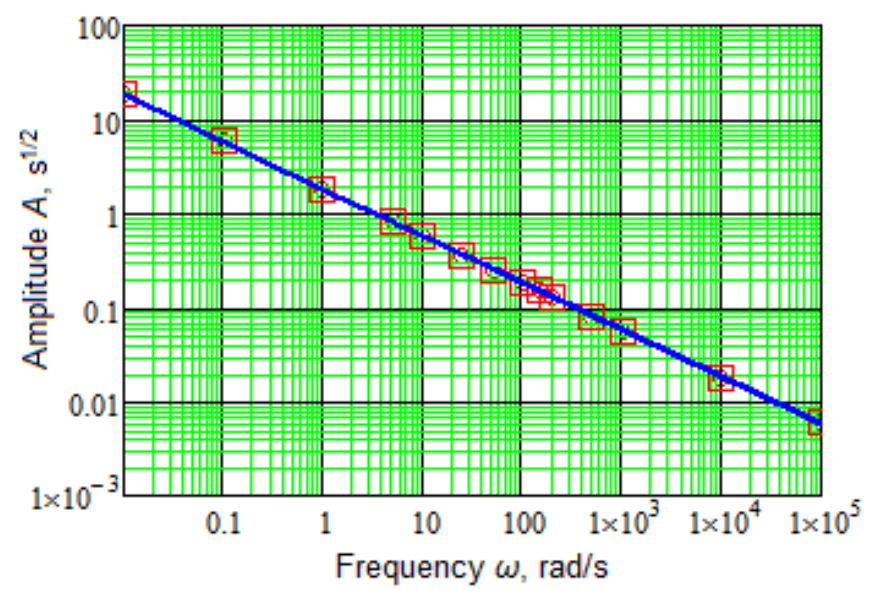

Fig. 1. Comparison of the theoretical curve with the numerical calculation data

Thus, the complementary function (6) is determined by the following analytical dependence:

$$
i_{s}(t, \omega)=\frac{1.82}{\sqrt{\omega}} \sin \left(\omega t-\frac{\pi}{4}\right)
$$

Similar to the above procedure, the following expression for another complementary function can be defined:

$$
i_{c}(t, \omega)=\int_{0}^{t} \frac{\cos \omega \theta}{\sqrt{t-\theta}} d \theta \approx \frac{1.82}{\sqrt{\omega}} \sin \left(\omega t+\frac{\pi}{4}\right) .
$$




\subsection{Clarification of the complementary functions}

In the previous item, the Basset force was determined by applying the regression analysis to the tabulated amplitude-frequency response. This approach requires clarification both in terms of the dimensionless constant $c$ and the phase shift $\pi / 4$. Therefore, the approach to more accurately justify the proposed analytical dependencies for determining the Basset force is presented below.

Applying the following change of parameters in the formula for the complementary function $i_{s}(\omega, t)$

$$
\begin{aligned}
& \phi=\omega(t-\theta) ; \Leftrightarrow \theta=t-\phi / \omega ; d \theta=-d \phi / \omega ; \\
& \omega \theta=\phi_{0}-\phi ; \theta=0: \phi=\omega t=\phi_{0} ; \theta=t: \phi=0
\end{aligned}
$$

allows rewriting equation (6) in the following form:

$$
i_{s}(t, \omega)=\int_{0}^{t} \frac{\sin \omega \theta}{\sqrt{t-\theta}} d \theta=\frac{1}{\sqrt{\omega}}\left(i_{2} \sin \phi_{0}-i_{1} \cos \phi_{0}\right),
$$

where the following functions are introduced:

$$
i_{1}\left(\phi_{0}\right)=\int_{0}^{\phi_{0}} \frac{\sin \phi}{\sqrt{\phi}} d \phi ; i_{2}\left(\phi_{0}\right)=\int_{0}^{\phi_{0}} \frac{\cos \phi}{\sqrt{\phi}} d \phi .
$$

Decomposition of these integral expressions into the following infinite power series $[20,21]$ with respect to the dimensionless parameter $\varphi_{0}$ allows writing:

$$
\begin{aligned}
& i_{1}\left(\phi_{0}\right)=\int_{0}^{\phi_{0}} \frac{1}{\sqrt{\phi}} \sum_{n=1}^{\infty} \frac{(-1)^{n+1} \phi^{2 n-1}}{(2 n-1) !} d \phi=\sum_{n=1}^{\infty} \frac{(-1)^{n+1}}{(2 n-1) !} \frac{\phi_{0}^{2 n-\frac{1}{2}}}{2 n-\frac{1}{2}} \\
& i_{2}\left(\phi_{0}\right)=\int_{0}^{\phi_{0}} \frac{1}{\sqrt{\phi}} \sum_{n=1}^{\infty} \frac{(-1)^{n+1} \phi^{2 n-2}}{(2 n-2) !} d \phi=\sum_{n=1}^{\infty} \frac{(-1)^{n+1}}{(2 n-2) !} \frac{\phi_{0}^{2 n-\frac{3}{2}}}{2 n-\frac{3}{2}} .
\end{aligned}
$$

The proposed formula (7) considering the expression (8) for the complementary function $i_{s}$ is a special case of the following general dependence:

$$
i_{s}(t, \omega)=\frac{c(t)}{\sqrt{\omega}} \sin [\omega t-\psi(t)],
$$

where $c(t), \psi(t)$ - dimensionless time and phase functions, respectively, which are determined by comparing the expressions (15) and (18): 


$$
\left\{\begin{array}{l}
c(t) \sin \psi(t)=i_{1}\left(\phi_{0}\right) ; \\
c(t) \cos \psi(t)=i_{2}\left(\phi_{0}\right) .
\end{array}\right.
$$

Thus, using the basic trigonometric correlations, it can be obtained:

$$
\begin{aligned}
& c\left(\phi_{0}\right)=\sqrt{i_{1}^{2}\left(\phi_{0}\right)+i_{2}^{2}\left(\phi_{0}\right)} ; \\
& \psi\left(\phi_{0}\right)=\operatorname{arctg}\left[i_{1}\left(\phi_{0}\right) / i_{2}\left(\phi_{0}\right)\right] .
\end{aligned}
$$

Due to the fact that both of the dimensionless time function $c\left(\varphi_{0}\right)$ and the phase function $\psi\left(\varphi_{0}\right)$ deviate from their mean values, the averaging procedure can be applied:

$$
\bar{c}=\lim _{\varphi \rightarrow \infty} \frac{1}{\varphi} \int_{0}^{\varphi} c\left(\phi_{0}\right) \approx 1.82 ; \bar{\psi}=\lim _{\varphi \rightarrow \infty} \frac{1}{\varphi} \int_{0}^{\varphi} \psi\left(\phi_{0}\right)=\frac{\pi}{4},
$$

which coincides with the previous expressions (12), (13).

\section{Results}

\subsection{Evaluation of the Basset force}

The motion of a dropped fluid-dispersed flow in a plane channel is considered. The flow is bounded by two walls along the flow. The first one is stationary, and the second one monoharmonically oscillates with the amplitude value of vibration velocity $a[\mathrm{~m} / \mathrm{s}]$, vibration frequency $\omega_{0}[\mathrm{rad} / \mathrm{s}]$, and wavelength $L=2 \pi / \lambda[\mathrm{m}]$. The design scheme is presented in Figure 2.

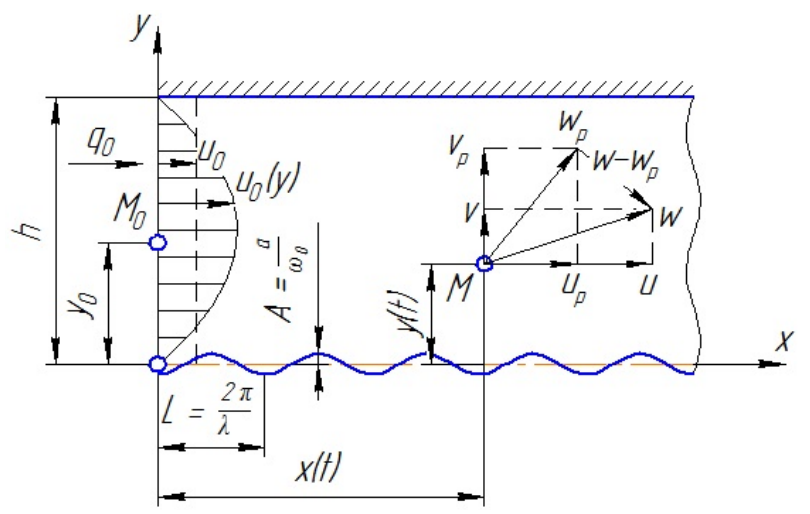

Fig. 2. The design scheme

To determine the Basset force, it is necessary to take the expressions for the acceleration components for a particle in the flow [22]: 


$$
\left\{\begin{array}{l}
\frac{d u}{d t}=\frac{6 \kappa_{1} a}{\lambda h}\left[\omega_{0} \cos \omega_{0} t-\frac{\omega_{0}-\lambda u_{0}}{2} \cos \left(\omega_{0}-\lambda u_{0}\right) t-\frac{\omega_{0}+\lambda u_{0}}{2} \cos \left(\omega_{0}+\lambda u_{0}\right) t\right] \\
\frac{d v}{d t}=\frac{\kappa_{2} a}{2}\left[-\left(\omega_{0}-\lambda u_{0}\right) \sin \left(\omega_{0}-\lambda u_{0}\right) t+\left(\omega_{0}+\lambda u_{0}\right) \sin \left(\omega_{0}+\lambda u_{0}\right) t\right]
\end{array}\right.
$$

where: $h$ - channel width $[\mathrm{m}], u_{0}$ - inlet velocity $[\mathrm{m} / \mathrm{s}], \lambda$ - wave parameter $\left[\mathrm{m}^{-1}\right]$, $\kappa_{1}, \kappa_{2}$ - dimensionless coefficients.

Particularly, for relatively small wavelengths $\left(\lambda>>\omega_{0} / u_{0}\right)$ :

$$
\left\{\begin{aligned}
f_{B x}= & 1.82 C_{B} \frac{6 \kappa_{1} a}{\lambda h}\left\{\sqrt{\omega_{0}} \sin \left(\omega_{0} t+\frac{\pi}{4}\right)-\frac{\sqrt{\lambda u_{0}-\omega_{0}}}{2} \sin \left[\left(\lambda u_{0}-\omega_{0}\right) t-\frac{\pi}{4}\right]-\right. \\
& \left.-\frac{\sqrt{\lambda u_{0}+\omega_{0}}}{2} \sin \left[\left(\lambda u_{0}+\omega_{0}\right) t+\frac{\pi}{4}\right]\right\} ; \\
f_{B y}= & 1.82 C_{B} \frac{\kappa_{2} a}{2}\left\{-\sqrt{\lambda u_{0}-\omega_{0}} \sin \left[\left(\lambda u_{0}-\omega_{0}\right) t+\frac{\pi}{4}\right]+\right. \\
& \left.+\sqrt{\omega_{0}+\lambda u_{0}} \sin \left[\left(\lambda u_{0}+\omega_{0}\right) t-\frac{\pi}{4}\right]\right\} .
\end{aligned}\right.
$$

In an extreme case $\left(\lambda>>\omega_{0} / u_{0}\right)$, and considering the identities for the transformation of the products of trigonometric functions into their sums, the following expressions for the components of the specific Basset force can be obtained:

$$
\left\{\begin{array}{l}
f_{B x}=-1.82 C_{B} \frac{6 \kappa_{1} a}{\lambda h} \sqrt{\lambda u_{0} / 2} \sin \lambda u_{0} t \\
f_{B y}=-1.82 C_{B} \kappa_{2} a \sqrt{\lambda u_{0} / 2} \cos \lambda u_{0} t .
\end{array}\right.
$$

In this case, the maximum value of the Basset force

$$
f_{B}^{\max }=\max \left(\sqrt{f_{B x}^{2}+f_{B y}^{2}}\right)=1.82 C_{B} a \sqrt{\left[\left(6 \kappa_{1} / \lambda h\right)^{2}+\kappa_{2}^{2}\right] \lambda u_{0} / 2} .
$$

The analysis of the obtained dependencies shows that the Basset force is directed toward the concavities of the particle's trajectory in a flow that enforces the particle to oscillate near the local pressure zones. Additionally, the Basset force periodically changes the direction with the frequency $\pi /\left(\lambda u_{0}\right)$ and decreases when particles are removed from the oscillating wall. Particularly, considering the values of dimensionless parameters near the oscillating wall are $\kappa_{1}=0$ and $\kappa_{2}=1$, its maximum value is determined by the following expression:

$$
f_{B 0}=\left.f_{B}^{\max }\right|_{y=0}=1.82 C_{B} a \sqrt{\lambda u_{0} / 2} .
$$


The angle $\theta$ of the Basset force deviation from the direction of the internal normal to the oscillating wall is determined by the following dependence:

$$
\operatorname{tg} \theta=\frac{f_{B x}}{f_{B y}}=\frac{6}{\lambda h} \frac{\kappa_{1}}{\kappa_{2}} \operatorname{tg} \lambda u_{0} t .
$$

This angle is equal to $0^{\circ}$ near the oscillating wall and equal to $90^{\circ}$ near the stationary wall (Fig. 3b).

a)

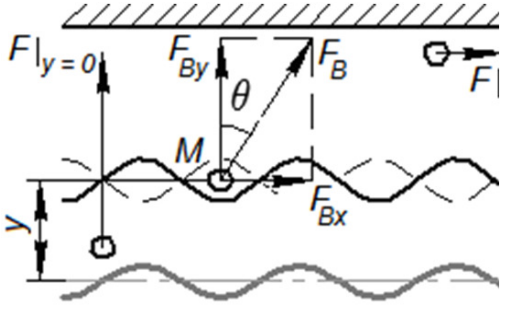

b)

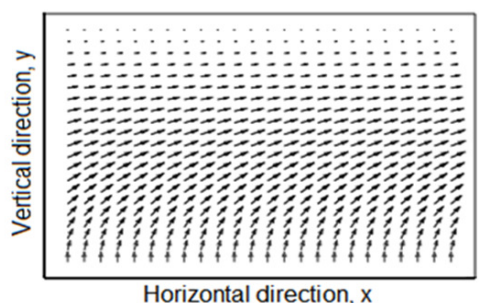

Fig. 3. The Basset force (a) acting on a particle at a given time, and its vector field (b)

\subsection{Impact of the Basset force on a particle}

Additionally, a relationship between the specific Basset force acting on a particle and flow acceleration in related point can be established. For the case of $\lambda>>\omega_{0} / u_{0}$, the components of the flow acceleration become simplified:

$$
\left\{\begin{array}{l}
a_{x}=\frac{6 \kappa_{1} a}{\lambda h} \lambda u_{0} \sin \omega_{0} t \sin \lambda u_{0} \\
a_{y}=\kappa_{2} a \lambda u_{0} \sin \omega_{0} t \cos \lambda u_{0}
\end{array}\right.
$$

and the maximum acceleration is equal to

$$
a_{\max }=\max \left(\sqrt{a_{x}^{2}+a_{y}^{2}}\right)=\lambda u_{0} \sqrt{\left[\left(6 \kappa_{1} / \lambda h\right)^{2}+\kappa_{2}^{2}\right]} .
$$

Comparing expressions (25) and (29) considering the dependence (2) the following expression can be found:

$$
F_{B}^{\max }=\frac{1.82 C_{B} a}{\sqrt{2 \lambda u_{0}}} m_{p} a_{\max } .
$$

The impact of the Basset force on the particles' dynamic in a fluid-dispersed flow can be evaluated by the dimensionless parameter as the ratio of the maximum components of the specific Basset force to the corresponding components of the vibration force. Particularly, considering the parameter $\beta$, the following expression for the impact of the Basset force on a particle is proposed: 


$$
C r_{B}=\frac{2 C_{B}}{\beta} \sqrt{\lambda u_{0}}=d_{p} \sqrt{\frac{\lambda u_{0}}{\pi v}} .
$$

Thus, the Basset force affects the larger particles. Moreover, the impact of the Basset force on particles of the disperse phase can be increased by reducing the vibration wavelength.

\section{Conclusions}

Thus, the analytical dependencies for determining the Basset force acting on particles of the dispersed phase in multiphase flow is proposed. The corresponding expressions are substantiated theoretically using both regression analysis and extending the complementary functions to infinite power series.

As a result of the numerical simulation, the Basset vector field is obtained. Moreover, the analysis of the obtained analytical dependencies shows that this force is directed towards the concavities of particles' trajectories in a flow. This fact proves the oscillations of particles relative to the local pressure zones. It has been additionally determined that the Basset force periodically changes its direction with the frequency $\pi /\left(\lambda u_{0}\right)$, and decreases when particles are removed from the vibrating wall.

A dimensionless criterion is proposed for determining the impact of the Basset force on particles of the dispersed phase in the flow with superimposed vibrations. As a result, it is determined that the Basset force affects particles of greater diameter, and the degree of its influence increases with a decrease of the vibration wavelength. These facts are the scientific foundation for ensuring the selective separation of multicomponent geterogeneous systems.

\section{Acknowledgements}

The article was funded by the Ministry of Education and Science of Ukraine within the project "Development and Implementation of Energy Efficient Modular Separation Devices for Oil and Gas Purification Equipment" (No. 0117U003931).

Experimental results were obtained within the project "Identification of Parameters for Technological Equipment using Artificial Neural Networks" funded by the National Scholarship Programme of the Slovak Republic.

\section{References}

[1] Gubaidullin, D.A., Osipov, P.P., \& Zakirov, A.N. (2014). Impact of Basset force on threshold values of particle drag coefficient and density parameter in standing sinusoidal wave. Journal of Physics: Conference Series, 567, 012018.

[2] Fan, F., Yang, X., \& Kim, C.N. (2013). Direct simulation of inhalable particle motion and collision in a standing wave field. Journal of Mechanical Science and Technology, 27(6), 1707-1712. 
[3] Gubaidullin, D.A., Osipov, P.P., \& Nasyrov, R.R. (2018). Influence of the drag coefficient of particles on their distribution in a two-dimensional acoustic resonator. Journal of Engineering Physics and Thermophysics, 91(3), 688-695.

[4] Wang, S., Allen, J.S., \& Ardekani, A.M. (2017). Unsteady particle motion in an acoustic standing wave field. European Journal of Computational Mechanics, 26(1-2), 115-130.

[5] Doughty, T.A., Belle-Isle, A.W., \& Pendowski, N. (2017). Experimental validation of nonlinear model tracking with varying conditions. Topics in Modal Analysis \& Testing, 10, 139-162.

[6] Brouwers, J.J.H. (2010). Langevin equation of a fluid particle in wall-induced turbulence. Theoretical and Mathematical Physics, 163(2), 677-695.

[7] Barjona, M., \& da Silva, C.B. (2017). Kolmogorov's Lagrangian similarity law revisited. Physics of Fluids, 29, 105106.

[8] Verhas, J. (2004). Onsager's reciprocal relations and some basic laws. Journal of Computational and Applied Mechanics, 5(1), 157-163.

[9] La Porta, A., Voth, G.A., Crawford, A.M., Alexender, J., \& Bodenschatz, E. (2001). Fluid particle accelerations in fully developed turbulence. Annual Review of Fluid Mechanics, 409, 1017-1019.

[10] Dostoglou, S. (2017). Statistical hydrodynamics and related problems in spaces of probability measures. AIP Conference Proceedings, 1907, 020004.

[11] Ilinskii, Y.A., Zabolotskaya, E.A., \& Hamilton, M.F. (2012). Acoustic radiation force on a sphere in tissue. AIP Conference Proceedings, 1474, 255-258.

[12] Sapozhnikov, O.A. (2013). Radiation force of an arbitrary acoustic beam on an elastic sphere in a fluid. The Journal of the Acoustical Society of America, 133(2), 661-676.

[13] Doinikov, A.A. (2002). Translational motion of a spherical bubble in an acoustic standing wave of high intensity. Physics of Fluids, 14, 1420.

[14] Steinberg, P. (2005). Landau hydrodynamics and RHIC phenomena. Acta Physica Hungarica, 24(1-4), 51-57.

[15] Sklabinskyi, V., Liaposhchenko, O., Pavlenko, I., Lytvynenko, O., \& Demianenko, M. (2019). Modelling of liquid's distribution and migration in the fibrous filter layer in the process of inertial-filtering separation. Advances in Design, Simulation and Manufacturing, DSMIE 2018, Lecture Notes in Mechanical Engineering, 489-497.

[16] Xu, S., \& Nadim, A. (2015). Three models for rectilinear particle motion with the Basset history force. Electronic Journal of Differential Equations, 104, 1-19.

[17] Liaposchenko, O., Pavlenko, I., \& Nastenko, O. (2017). The model of crossed movement and gas-liquid flow interaction with captured liquid film in the inertial-filtering separation channels. Separation and Purification Technology, 173, 240-243.

[18] Shen, C., Wang, W., He, S., \& Xu, Y. (2018). Numerical and experimental comparative study on the flow-induced vibration of a plane gate. Water, 10(11), 1551.

[19] Liaposhchenko, O.O., Sklabinskyi, V.I., Zavialov, V.L., Pavlenko, I.V., Nastenko, O.V., \& Demianenko, M.M. (2017). Appliance of inertial gas-dynamic separation of gas-dispersion flows in the curvilinear convergent-divergent channels for compressor equipment reliability improvement. IOP Conference Series: Materials Science and Engineering, 233(1), 012025.

[20] Kirsh, V.A. (2016). Stokes flow past periodic rows of porous cylinders. Theoretical Foundations of Chemical Engineering, 40(5), 465-471.

[21] Kinjal, P., Mehta, M.N., \& Patel, T.R. (2011). The power series solution of fingering phenomenon arising in fluid flow through homogeneous porous media. Applications and Applied Mathematics, 6(2), 497-509.

[22] Pavlenko, I.V., Liaposhchenko, O.O., Sklabinskyi, V.I., Ivanov, V.O., \& Gusak, O.G. (2018). Hydrodynamic features of gas-liquid flow movement in a separation device plane channel with an oscillating wall. Problemele Energeticii Regionale, 3(38), 62-70. 